Open Peer Review on Qeios

\title{
The Synthetic Scaffolds for Ventral Hernia Repair: Perspectives for Regenerative Surgery-Systematic Review
}

Leo Licari

Funding: The author(s) received no specific funding for this work.

Potential competing interests: The author(s) declared that no potential competing interests exist.

\section{Abstract}

Ventral hernia $(\mathrm{VH})$ frequently affects patients after abdominal surgery. The use of a mesh is often recommended. Different materials are described, from synthetic non-resorbable meshes to biological meshes. New generation meshes, also named scaffolds, aim to combine the advantages of both materials. The aim of this review is to provide an overview of the cytological, histological, biomechanical, and clinical outcomes of the use of the newest resorbable synthetic scaffolds in VH repair, based on experimental studies in a pre-clinical setting. A systematic review was conducted according to the Preferred Report- ing Items for Systematic Reviews and Meta-Analyses (PRISMA) guidelines and to the Assessing the Methodological Quality of Systematic Reviews (AMSTAR) guidelines. Only experimental studies were included. Outcome parameters were building technique, in vitro cytocompatibility, in vivo histocompatibility, biomechanical analysis, and clinical outcomes. The articles included were nine. The total number of cases treated was 257. Materials analyzed included electrospun silk fibroin (SF)/poly (3hydroxybutyrate-co-3-hydroxyvalerate) (PHBV) hybrid scaffolds, biodegradable polyester poly- $\varepsilon$ caprolactone $(P C L)$ in the form of nanofibers, biodegradable mesh in poly-4-hydroxybutyrate (P4HB), nanofibrous polylactic acid (PLA) scaffold with a polypropylene (PP) material to generate a sandwich-like mesh, the collagen sponge (CS) group, the hybrid scaffold (HS) containing CS and poly-L-lactide (PLLA), and the hybrid scaffold (HS) + bone marrow (HSBM). Resorbable synthetic scaffolds are new, safe, surgical materials for the treatment or prevention of ventral hernia in animal models. Scaffolds should be tested in a contaminated surgical field for emergency use. Rigorous schematic indications for data collection are needed to improve the quality of the data in order to definitively clarify the pathway involved in inflammatory induced response.

Indian Journal of Surgery https://doi.org/10.1007/s12262-021-03205-2

\section{Introduction}

Ventral hernia repair (VHR) represents one of the major issues in the surgical field, and it represents the most com- mon complication after abdominal surgery $[1,2]$. 
The ideal mesh for VHR should withstand the implantation process as well as the patient's postoperative activities. In addition, the optimal mesh should minimize complications connected with the wound-healing process [3-6].

The aim of the study is (1) to analyze the data in the lit- erature concerning the newest synthetic absorbable scaffolds for VHR in a pre-clinical setting. The second goal is (2) to collect all the information about the cytologic, histologic, and biomechanical characteristics of those scaffolds.

\section{Methods}

We conducted a systematic review according to the Preferred Reporting Items for Systematic Reviews and Meta-Analyses (PRISMA) guidelines and to the Assessing the Methodological Quality of Systematic Reviews (AMSTAR) guidelines. A systematic search was performed in the MEDLINE, as well as in Embase, Web of Science, Scopus, PubMed Pub- lisher, Google Scholar, and the Cochrane Library, with a search period until November 2019. Searches were adapted to each database and carried out using the specific controlled vocabulary of each database, if available, as well as free-text words. The search included the words "scaffold," “hernia," "abdominal," “wall," and "reconstruction."

Two reviewers (L.L. and G.S.) independently evaluated the identified records. Inclusion criteria were as follows: experimental studies, investigating resorbable synthetic meshes, with or without comparison of synthetic and/or biological meshes. English language restriction of the articles was set. Clinical studies and studies that investigated only biological or non-resorbable synthetic meshes were excluded. The following outcome measurements were assessed: in vitro cytocompatibility, in vivo histocompatibility, and biomechanical analysis in animal models. The data presented in this review were directly abstracted from the origi- nal articles. No statistical analyses were performed.

\section{Results}

A total of 53 articles were considered relevant. After an analysis of the full text of these articles, nine were included in the review (Fig. 1). The reasons for exclusion can be found in the PRISMA flow diagram. The total number of cases treated was 257 and, because of the nature of the data presented, 149 were rats, 27 rabbits, 27 minipigs, and 54 Yucatan pigs. One paper examined the feasibility of using electrospun silk fibroin (SF)/poly (3-hydroxybutyrate-co-3-hydroxyvalerate) (PHBV) hybrid scaffolds [7]. Three papers examined a bio- degradable polyester poly- $\varepsilon$-caprolactone $(P C L)$ in the form of nanofibers as a scaffold for fascia healing [8-10]. PCL scaffolds were combined with polypropylene (PP) material to generate a sandwich-like mesh. Results were compared with control groups (no mesh, PCL alone, PP/PCL enriched with growth factors (GF), PP alone). Three papers examined a biodegradable mesh in poly-4-hydroxybutyrate (P4HB) [11-13]. One paper examined a mesh composed by nanofi- brous polylactic acid (PLA) and polypropylene (PP) [14] combining a nanofibrous PLA scaffold with a PP material to generate a sandwichlike mesh, with the peritoneum side of the mesh coated with an extra layer of PLA to prevent adhesion. One paper examined and compared three scaf- folds: the collagen sponge (CS) group; the hybrid scaffold (HS) containing CS and poly-L-lactide (PLLA); the hybrid scaffold (HS) + bone marrow named HSBM group [15]. 
In the following sections, the features of each scaffold and outcome parameters are given.

\section{Building Technique}

Electrospinning is a convenient technique to fabricate nanofiber scaffolds with a small diameter, large specific surface area, and high surface area volume ratio, which can be conducive to cell adhesion and proliferation, and promote the adsorption and release of active cells and growth factors; therefore, the biological properties of nanofiber scaffolds will be greatly improved [16]. P4HB mesh is made from a biosynthetic polymer monofilament fiber [11] that is knit into a flat sheet configuration [12].

\section{In Vitro Cytocompatibility}

Concerning the SF/PHBV scaffold, the morphology of fibroblasts cultured on it was observed directly by scan- ning electron microscopy (SEM). The morphology of the fibroblasts indicated that the SF/PHBV nanofiber scaffolds could support cell adhesion and migration and be nontoxic to cellular entities. Three scaffolds with different compo- nent ratios were analyzed: 25/75, 50/50, 75/25. The results indicated that the SF/PHBV nanofiber scaffold with a 75/25 ratio was more suitable for fibroblast adhesion and growth. The 75/25 ratio shows higher cell doming at days 5 and 7 . Moreover, the fibroblasts cultured on SF/PHBV nanofiber scaffolds with a 75/25 ratio could significantly increase the gene expression of Transforming Growth Factor- $\beta 1$ (TGF- $\beta 1)$ and Collagen I $(p<0.05)$ [7].

Concerning the PP/PCL and PCL scaffolds, samples were treated with growth factors (GFs)[8, 9] that were bound to the scaffold by $12 \mathrm{~h}$ of incubation in a phosphate-buffered saline solution, which contained 200 ng/mL Insulin Growth Factor-I (IGF-I), 40 ng/mL basic Fibroblast Growth Factors (FGF), and 4 ng/mL Transforming Growth Factor- $\beta 2$ (TGF- $\beta 2$ ). These GFs were used to stimulate proliferation, glycosaminoglycan, and collagen synthesis [8]. The characteristics of fibroblasts cultured on the scaffolds composed by PCL were observed directly by scanning electron microscopy (SEM) and confocal microscope [8-10]. Confocal micros- copy images of the PP/PCL and PCL samples showed better initial adhesion and, subsequently, an improved prolifera- tion rate of fibroblasts compared to PP meshes. SEM images showed sub-confluent layers on the nanofiber-containing scaffolds in contrast to the PP mesh, where the cells were well spread, but very scarce due to its low surface-to-volume ratio.

Scanning electron microscopy images of P4HB mesh specimens after in vitro incubation in $3 \mathrm{~mol} / \mathrm{L} \mathrm{HCl}$ at $37{ }^{\circ} \mathrm{C}$ demonstrated visibly intact mesh through $32 \mathrm{~h}$ of incubation, bulk structural changes by $72 \mathrm{~h}$, and significant bulk degra- dation by $96 \mathrm{~h}$ [11].

Concerning the PLA/PP mesh, the morphology of fibro- blasts cultured on it was observed directly by SEM. The PLA/PP mesh showed a better cell proliferation rate than the control group (PP and polyethylene (PE) alone) after 1 and 3 days. There was no significant difference in cell viability among the materials on day 5. At the first two time points, the PLA/PP mesh stimulated cell growth better than the control groups. The

E-cadherin expression in mesothelial cells was downregulated after stimulation by TGF- $\beta 1$, while the expression of $\alpha$-Smooth Muscle Actin ( $\alpha$-SMA) and collagen I was upregulated. The mesothelial cells were transformed to fibroblasts via the TGF- $\beta 1 /$ Smad pathway. The immunohistochemical study revealed that cells adherent to the mesh with fibroblast or polygonal shapes or giant-cell morphologies were all 
positively stained by anticytokeratin and antivimentin antibodies, which confirmed that the cul- tured cells had a mesothelial phenotype [14].

Concerning CS/HS/HSBM scaffolds, no data are provided in the study with regard to in vitro cytocompatibility [15].

\section{In Vivo Histocompatibility}

Concerning the SF/PHBV scaffold, in vivo tissue regenera- tion was tested on rats. The exact position of the scaffold in the abdominal wall is not shown in the study. Under hema- toxylin and eosin (H\&E) staining, more granulation, connec- tive tissue depositions, and microvascular angiogenesis were detected in repaired subcutaneous superficial muscle tissues subjected to SF/PHBV nanofiber scaffolds, which clearly differed from irregular fibrous tissues, fibrinous debris, and microvascular ingrowth of the control group that was treated without implantation after 7 and 15 days. Furthermore, there was marked hyperplasia of fibroblast accompanied by increased deposition of Extra Cellular Matrix (ECM) in the SF/PHBV-treated group relative to that of the control group treated without implantation. Under Sirius red staining collagen fibers, the SF/PHBV nanofiber-treated group was more aligned, formed, and better interwoven relative to that of the control group and presented more hierarchical and brighter bundle structures than those from the control group treated without implantation at 7 and 15 days [7].

Concerning the PCL scaffold, in vivo tissue regeneration was tested on rabbits [8, 9] and minipigs [10]. Scaffolds were placed in onlay position. There were more $\alpha$-smooth-muscle actin-positive cells in the area of the healing incision in all samples containing PCL nanofibers than in the controls. Significant differences in the density of the micro-vessel profiles were observed between the groups. Samples con- taining PCL nanofibers had a greater density of micro-vessel profiles than the control groups. The density was clearly highest in the portion of samples with mesh functionalized with PCL nanofibers enriched with adhered GF. The high- est fraction of collagen was observed in samples with PCL nanofibers enriched with adhered GF, followed by samples functionalized with PCL nanofibers. The presence of PCL nanofibers and GF seemed to increase granulomatous infil- tration and vascularization of the healing tissue, because the remnants of nanofibers were surrounded by granulomatous leukocyte-rich connective tissue at the end of week 6 after implantation. Tissue samples with heavier granulomatous infiltration also contained more blood vessels and a higher fraction of vascular smooth muscle and myofibro- blasts [9]. The study by East et al. [10] revealed that the incision area of the PP/PCL and PCL group contained a greater area fraction of actinpositive myofibroblasts than the Prolene group (Mann-Whitney $U$-test: $p=0.002$ ). The density of the micro-vessel profiles within the healing inci- sion was greater in the Prolene group than in the PP/PCL group (Mann-Whitney $U$-test: $p=0.018$ ). The amount of collagen type I depended more on the total amount of the myofibroblasts in the Prolene group. The composite material was less infiltrated with inflammatory cells than the poly- propylene implant, collagen fibrils were more aligned, and the scar contained less fat tissue [10].

The study by Martin et al. [11] demonstrated the pres- ence of a moderate chronic host inflammatory response surrounding the $\mathrm{P} 4 \mathrm{HB}$ mesh knots and monofilaments at $8,16,32,48$, and 72 weeks post- 
implantation. The mesh was positioned in the preperitoneal space of Yucatan pigs. This response primarily consisted of macrophages, lymphocytes, and occasional giant cells.

Scott et al. [13] demonstrated the host inflammatory response to P4HB scaffold when differently positioned in the intraperitoneal and retro-muscular placement. The experimental model was the Yucatan pig. In both condi- tions, P4HB mesh was associated with mild inflammation and minimal to mild fibrosis and neovascularization, which did not change significantly between 12 and 24 weeks.

Concerning the PLA/PP scaffold, in vivo tissue regenera- tion was tested on rats. The histopathology results showed that the inflammatory response was milder with the new mesh than with the control group. At 4 weeks post-implantation, the meshes contained substantial numbers of host cells, as well as a well-defined band of scaffold with minimum cell infiltration. At 8 weeks post-implantation, more host cells had infiltrated and reached into the scaffold, and ECM pro- teins had formed. At 12 weeks post-implantation, the prevalence of host cells was markedly reduced, and increased ECM deposition and vascularization were detected. The expression of collagen I was higher with the new type of mesh at each time point than with the other meshes. In contrast, the expression of collagen III was lower with the new type of mesh at each time point. A high ratio of collagen type $\mathrm{I} / \mathrm{III}$ is assumed to be an indicator of high-quality scar tissue (fibrosis) [14].

Concerning the CS/HS/HSBM scaffold, in vivo tissue regeneration was tested on rats. The CS was absorbed rap- idly in vivo, and therefore, a histological examination of the CS group was impossible. Histologically, H\&E staining revealed clearly visible vessels containing red blood cells in all animals in the HS and HSBM groups. The capillary den- sity was evaluated and graded using the method of Nowacki et al. The capillarity density score of the HSBM group was significantly higher than the HS group at 4, 8, and 16 weeks. At the center of the implant, skeletal muscle cells were not recognized in either the H\&E or desmin staining method. Regarding the border lesion, skeletal muscle cell replacement was apparent. Skeletal muscle cell replacement was observed at $40 \%$ at 4 weeks, $20 \%$ at 8 weeks, and $20 \%$ at 16 weeks, respectively, only in the HSBM group. Skeletal muscle cell replacement in the implant was only recognized in the HSBM group [15].

\section{Biomechanical Analysis}

Concerning the SF/PHBV scaffold, the study by Gong et al. showed the characteristics of the stress-strain curve and the data of breaking strength and elongation at the break of SF/ PHBV nanofibers. With reduced PHBV content, the fracture strength of the nanofibers decreased and the elongation at break increased. The SF/PHBV scaffold with a 75/25 ratio showed a breaking strength of 2.15 SD0.18 MPa with a 3.98\% SD0.43 elongation at the break [7].

Concerning PCL/PP scaffolds, biomechanical testing at 6 weeks post-implantation showed that the PCL nanofibers improved the biomechanical properties of the healed tissue, as evidenced by a higher average maximal strength force. This applied not only to the nanofibers in combination with a PP mesh, but also to the suture alone. The group treated with suture and PCL nanofibers with or without adhered GFs showed a modulus of elasticity comparable with that of the PP mesh, but with a higher average maximal strength 
force. This could reflect lower fibroblast proliferation around the PP mesh, which might be caused by a reduction in the mechanical signals that arise as the structural soft tissue fails.

In the study by Scott et al. [13], it was demonstrated that, in intraperitoneal placement, the P4HB mesh/repair strength at 12 weeks (298.4 SD24.7 N) was 44\% greater than the T0 strength of the mesh alone. Furthermore, the P4HB mesh/ repair strength at 24 weeks (275.8 SD59.6 N) was 33\% greater than the T0 strength of the mesh alone. However, it should be noted that this increase in strength relative to the T0 strength of the mesh alone represents the contribution of the abdominal wall, any newly formed tissue, and the mesh scaffold. The mesh/repair strength of P4HB was also significantly greater than the native abdominal wall (NAW) (61.5 SD16.7 N) at both 12 and 24 weeks ( $p<0.001$ in both cases). In retromuscular placement, the P4HB mesh/repair strength at 12 weeks (271.4 SD52.7 N) was 35\% greater than the T0 strength of the mesh alone. Furthermore, the P4HB mesh/repair strength at 24 weeks (317.1 SD85.6 $\mathrm{N})$ was $58 \%$ greater than the T0 strength of the mesh alone. However, the mesh/repair strength of P4HB was significantly greater than the NAW $(78.4 \mathrm{SD} 25.0 \mathrm{~N}$ ) at 12 and 24 weeks ( $p<0.001$ in both cases). Mechanical testing revealed that sites repaired with P4HB scaffolds had a similar mesh/repair strength and were both significantly stronger than NAW at 12 and 24 weeks. These results suggest that P4HB, with associated tissue ingrowth, augmented the strength of the NAW despite partial resorption of mesh fibers comprising these devices.

Concerning PLA/PP scaffold, the tensile properties of the meshes were calculated from their stress-strain curves. The tensile strength of the PLA/PP scaffold was 5.67 SD0.18 MPa. The stress of the PLA/PP scaffold was 32.73 SD0.48 N, which was suitable for a large hernia. At a stress of $16 \mathrm{~N}$, meshes should show a strain of at least $15 \%$ in horizontal stretching to achieve near-physiological proper- ties [14].

Concerning CS/HS/HSBM scaffolds, no data are pro- vided in the study with regard to biomechanical analysis [15].

\section{Discussion}

The review conducted shows an overview of the most recent discoveries with regard to the resorbable synthetic scaffolds that are currently experimented in a pre-clinical setting.

Ideally, the scaffold should offer the strength of traditional meshes and induce wound healing similarly to the native abdominal wall structure, integrating and resorbing over time [11].

Currently, it is still unclear in what way the inflamma- tory response induced by the scaffolds is able to guide the site-specific remodeling of fascia and muscle tissues. His- tologic analysis of the studies analyzed provided important data about the behavior of the different characters of inflammation.

Collagen should be considered an excellent material for creating scaffolds for tissue engineering thanks to its high affinity of cell adherence and growth-stimulating effects. This important characteristic is antagonized by the disad- vantage of the ready absorption of the collagen in vivo.

Suzuhigashi et al. [15] presented the results of their experimentation of a composite material of collagen sponge (CS) plus PLLA and of the hybrid scaffold composed of CS + PLLA + bone marrow, named HSBM. Their results should be summarized as follows: (1) a hybrid scaffold using a CS and PLLA was feasible for 
the abdom- inal hernia; (2) vascular formation in the HSBM group was clearly recognized compared with the HS group; (3) skeletal muscle cell replacement in the implant was only recognized in the HSBM group; and (4) the triad of scaffold, cell, and growth factor is fundamental for ideal biomaterials.

A significant increased response of type I collagen dep- osition has also been observed in the study groups that used SF/PHBV [7] scaffolds in PCL with the addition of growth factors [9] and in the PP/PLA group [14]. In the group of samples that underwent a PCL/PP-based com- posite implant, the large part of type I collagen was found in regions with a large and prominent density of microvessels, including capillaries [810].

Nanostructured PLA scaffolds are suitable for tissue reconstruction because of their advantages in cell adher- ence, proliferation, and migration. However, the poor mechanical strength of nanostructured PLA scaffolds limits their use in hernia repair. A combination with synthetic non-absorbable material is required [14].

Among the objectives of the surgical intervention for abdominal wall hernia repair are both anatomical and func- tional restoration and, above all, mechanical restoration.

The results provided by the study by East et al. [8] showed that the dynamic properties of healing midline laparotomy can be influenced by the implant used and that a mesh construct with zero mechanical strength act- ing purely as a bioscaffold can provide a stronger and more elastic abdominal wall compared to conventional hernia mesh. There is increasing evidence that many of the cur- rent hernia mesh implants may be over-engineered. Klinge et al. reported that some meshes show rupture forces of 40-100 N/cm, when in fact the forces acting on the abdominal wall may be as low as 4-16 N/cm [17]. Eliason et al. have demonstrated in vitro how these mesh materials deteriorate during repetitive loading [4].

\section{Conclusions}

The comprehension of the microscopic-induced modifica- tions by the scaffold to the abdominal wall anatomy leads to macroscopic evidence that the healing induced tissue has notable strength and biomechanical characteristics.

Randomized controlled trials and prospective registries in humans with a sufficiently long follow-up period should be encouraged to reveal the potential advantages of synthetic resorbable scaffolds in clinical practice.

\section{References}

1. Licari L, Guercio G, Campanella S et al (2019) Clinical and functional outcome after abdominal wall incisional hernia repair: evaluation of quality-of-life improvement and comparison of assessment scales. World J Surg 43(8):1914-1920

2. Salamone G, Licari L, Guercio G et al (2018) The abdominal wall hernia in cirrhotic patients: a historical challenge. World J Emerg Surg 13:35

3. Jacob BP, Ramshaw B (2013) The SAGES Manual of Her- nia Repair. Springer, New York, NY. https://doi.org/10.1007/ 978-1-4614-4824-2

4. Eliason BJ, Frisella MM, Matthews BD, Deeken CR (2011) Effect of repetitive loading on the mechanical 
properties of synthetic hernia repair materials. J Am Coll Surg 213(3):430-435

5. Kim SH, Turnbull J, Guimond S (2011) Extracellular matrix and cell signalling: the dynamic cooperation of integrin, proteoglycan and growth factor receptor. J Endocrinol 209(2):139-151

6. Ma Z, Kotaki M, Inai R, Ramakrishna S (2005) Potential of nanofiber matrix as tissue-engineering scaffolds. Tissue Eng 11(1-2):101-109

7. Gong W, Cheng T, Liu Q et al (2018) Surgical repair of abdominal wall defect with biomimetic nano/microfibrous hybrid scaffold. Mater Sci Eng C Mater Biol Appl 93:828-837

8. East B, Plencner M, Otahal M et al (2019) Dynamic creep prop- erties of a novel nanofiber hernia mesh in abdominal wall repair. Hernia 23(5):1009-1015

9. Plencner M, East B, Tonar Z et al (2014) Abdominal closure reinforcement by using polypropylene mesh functionalized with poly- $\varepsilon$-caprolactone nanofibers and growth factors for prevention of incisional hernia formation. Int J Nanomedicine 9:3263-3277

10. East B, Plencner M, Kralovic M et al (2018) A polypropyl- ene mesh modified with poly- $\varepsilon$-caprolactone nanofibers in hernia repair: large animal experiment. Int J Nanomedicine 13:3129-3143

11. Martin DP, Badhwar A, Shah DV et al (2013) Characterization of poly-4-hydroxybutyrate mesh for hernia repair applications. J Surg Res 184(2):766-773

12. Deeken CR, Matthews BD (2013) Characterization of the mechan- ical strength, resorption properties, and histologic characteris- tics of a fully absorbable material (Poly-4-hydroxybutyrate- PHASIX Mesh) in a porcine model of hernia repair. ISRN Surg 2013:238067

13. Scott JR, Deeken CR, Martindale RG, Rosen MJ (2016) Evalua- tion of a fully absorbable poly-4hydroxybutyrate/absorbable bar- rier composite mesh in a porcine model of ventral hernia repair. Surg Endosc 30(9):3691-3701

14. Xiaolong Y, Xiaoyan H, Bo W et al (2018) Ventral hernia repair in rat using nanofibrous polylactic acid/polypropylene meshes. Nanomedicine (Lond) 13(17):2187-2199

15. Suzuhigashi M, Kaji T, Nakame K et al (2016) Abdominal wall regenerative medicine for a large defect using tissue engineering: an experimental study. Pediatr Surg Int 32(10):959-965

16. Wang C, Wang J, Zeng L, et al (2019) Fabrication of electro- spun polymer nanofibers with diverse morphologies. Molecules. 24(5):834 gol. Neck Surg. 2006;132(3):293-8

17. Klinge U, Conze J, Limberg W et al (1996) Pathophysiology of the abdominal wall. Chirurg 67(3):229233 